\title{
JWST - The Even Bigger Time Machine
}

\section{Martin Topinka*t}

Dublin Institute of Advanced Studies, School of Cosmic Physics, 31 Fitzwilliam Place, Dublin,

Ireland

E-mail: martin.topinka@gmail.com

\begin{abstract}
After its launch, the James Webb Space Telescope (JWST) will become one of the most important space mission in the history. This mid-infrared telescope will benefit from the largest collecting area mirror ever sent to the space. A set of many various instruments will allow the scientists to explore, along many other interesting observations, the dawn of the Universe at high redshifts, to observe the very first stars and galaxies as well as to look at selected exoplanets. In this article three scientific tasks are highlighted: the JWST deep field; a search for lensed very distant Pop III stars; and follow-up observations of short gamma-ray bursts with the aim to detect a kilonova counterpart.
\end{abstract}

Frontier Research in Astrophysics - III (FRAPWS2018)

28 May - 2 June 2018

Mondello (Palermo), Italy

${ }^{*}$ Speaker.

${ }^{\dagger}$ Sincere thanks to Franco Giovannelli for the invitation! 


\section{Introduction}

The James Webb Space Telescope [1, 2], often presented as the next generation Hubble Space Telescope, is being constructed as a collaboration between NASA, European Space Agency (ESA) and the Canadian Space Agency (CSA). The project is lead by Goddard Space flight Centre, having the Northrop Grumman company as the prime constructor, while the Space Telescope Science Institute is responsible for the telescope operation. The involvement in the project gives the European side of the co-operation a guaranteed minimum of $15 \%$ of the observing time.

The design of the telescope covers the primary and the secondary mirror, the spacecraft bus and the sun shield among the instruments and detectors. The overall build is depicted in the Figure 1. With the segmented primary gold-coated mirror $6.6 \mathrm{~m}$ in diameter, made of 18 segments in Beryllium, enhanced with an active optic system, together with the bus and the sun shield of a size of a tennis court $(26 \mathrm{~m} \times 14 \mathrm{~m})$, JWST will become the largest space telescope mission ever launched.

The mission life time, mostly determined by the fuel for manoeuvring, is expected to be around 10 years, with the minimum engineered time of 5 years.

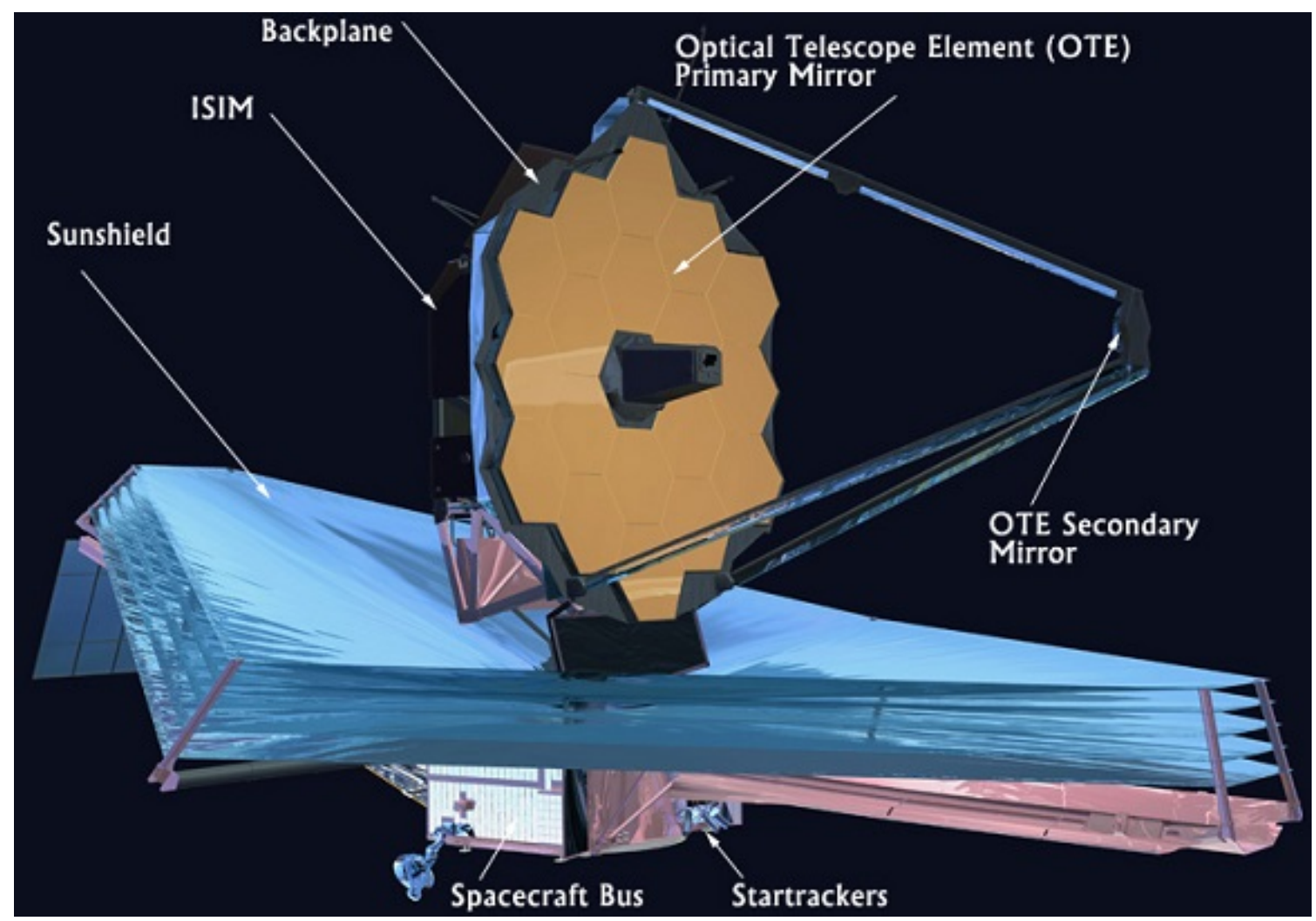

Figure 1: A concept of JWST with the primary mirror, the secondary mirror, the sun shield and the spacecraft bus and star-trackers. Credit: NASA.

\section{Instruments On-Board}

JWST will be an infra-red telescope, optimised for the wavelength range from $0.6 \mu \mathrm{m}$ to 
$28 \mu \mathrm{m}$ to be able to observe distant galaxies, "cool" objects and to peer into very dusty environments. The size of the mirror, the detector sensitivity and a low level of the telescope infra-red self background radiation will enable the telescope to gather a very faint signal from the near-infrared to the mid- infrared range of the spectrum. An angular resolution is similar to the HST one but in the near infrared. A 6.5-meter diameter primary mirror diffraction limited is at around 2 microns and provides a very good pointing stability.

To diminish the IR radiation background noise, the telescope will be deployed at the L2 point of the Earth-Sun system orbit, 1.5 million kilometres from the Earth. Thanks to the gravitational pull of the Earth-Sun system the period will be $\sim 12$ months. The orientation of the telescope and the geometry of the sun shield with respect to the Sun limits the viewing directions. The JWST field of regard extends from a solar elongation of $85^{\circ}$ to $135^{\circ}$ and changes over time as the observatory orbits the sun.

Due its size the deployment resembles an origami unfolding from the compact state in the cargo head of a launching rocket. JWST will "unpack" the solar array, the sun shield, the mirror and erect the secondary mirror on the way to L2, as seen in the Figure 2. The process will take two weeks from the launch.

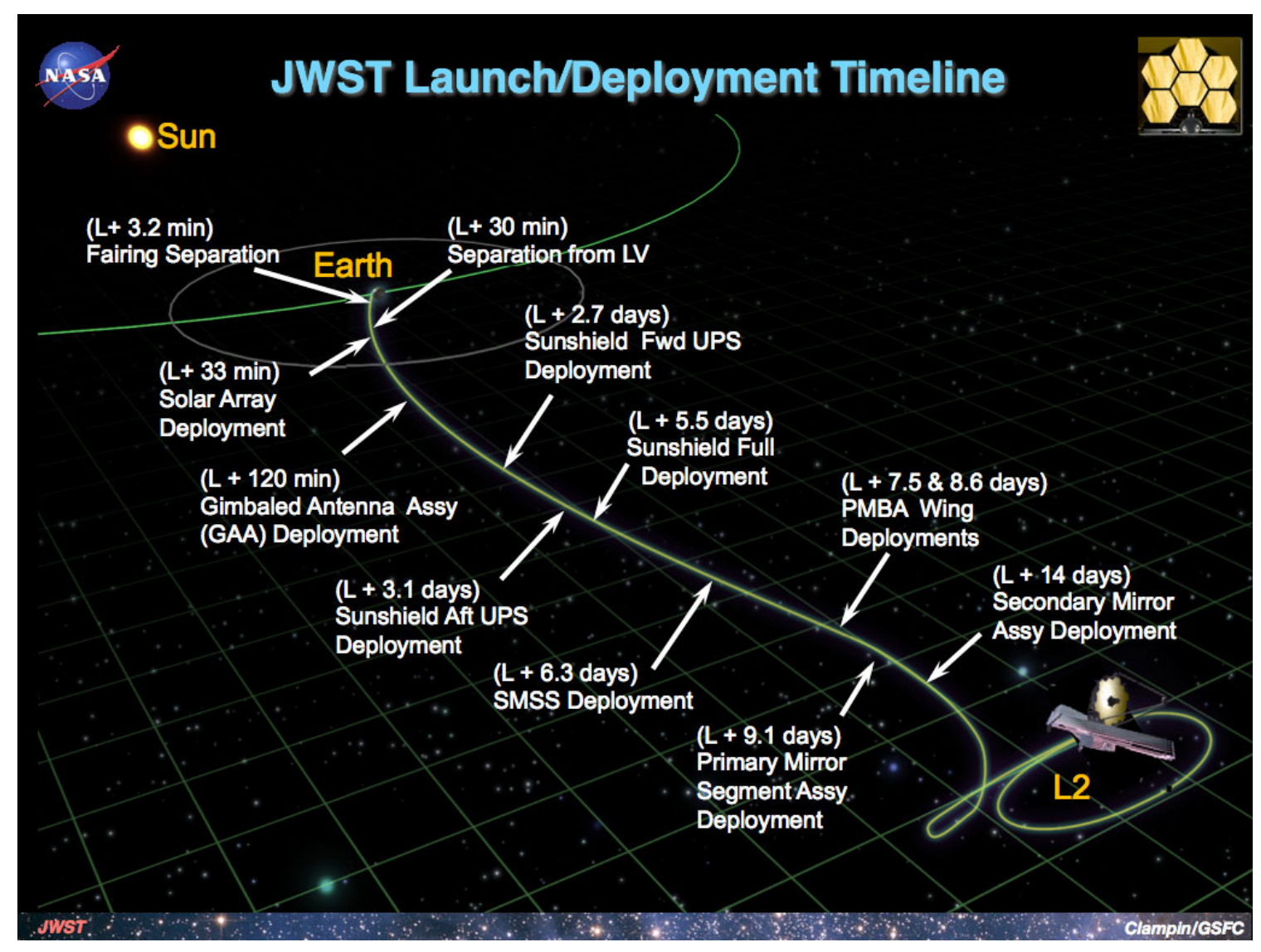

Figure 2: A depiction of the JWST deployment on its way to L2. Credit: NASA/GSFC.

JWST has four (or five, depends on the definition) main instruments on board [3]: NIRCam, NIRSpec, NIRISS and MIRI (and the fly guidance star tracker). Their field of views are not co- 
centric, their FOV orientations with respect to the telescope pointing axes are shown in the Figure 3.

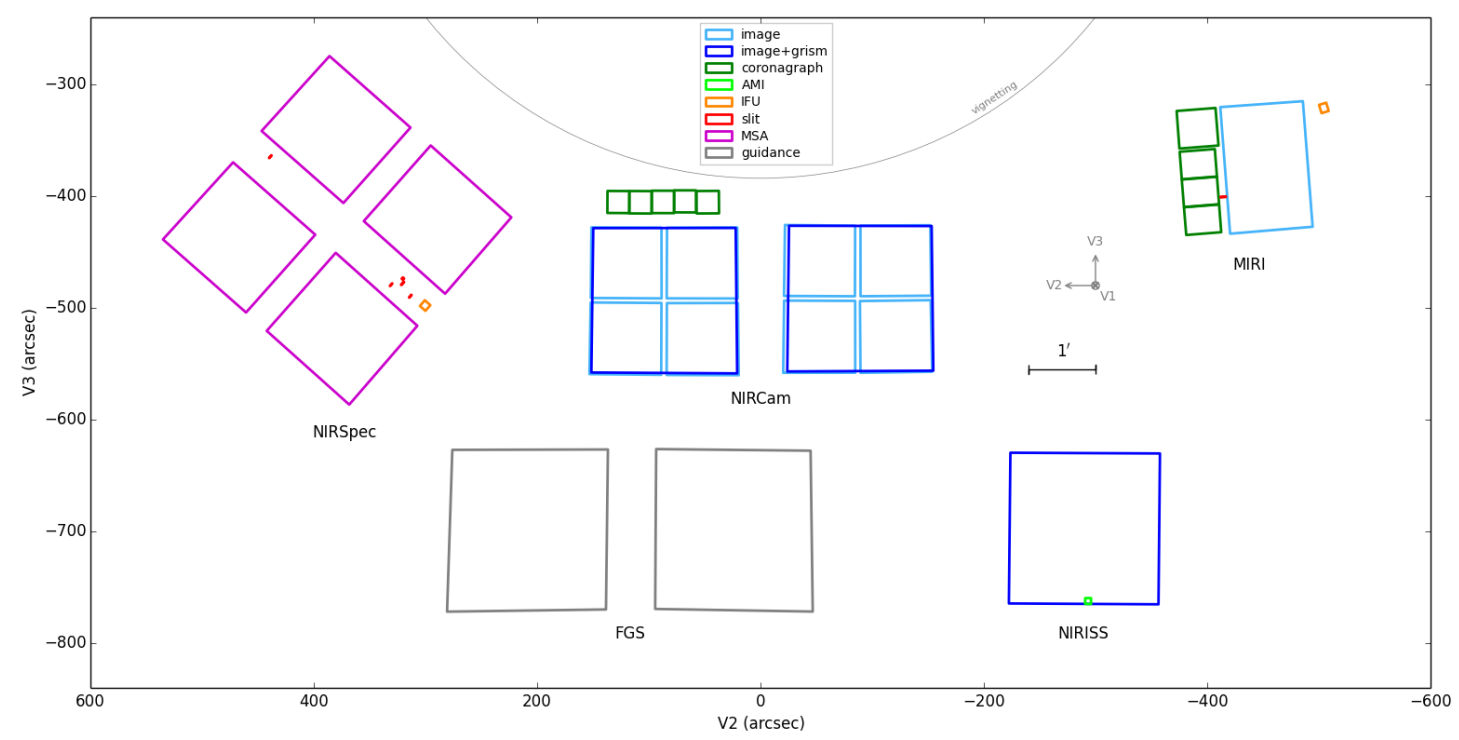

Figure 3: The JWST instrument FOVs are pointing towards the same direction, however, they are not cocentric. Parallel observations require a specific dither pattern. Credit: NASA/STScI.

The Near Infrared Camera (NIRCam) is JWST's primary imager that will cover the infrared wavelength range 0.6 to 5 microns. NIRCam is equipped with coronagraphs. The Near InfraRed Spectrograph (NIRSpec) will operate also over the wavelength range of 0.6 to 5 microns. The NIRSpec is designed to observe up to 100 objects simultaneously. It will be the first spectrograph in space that has this remarkable multi-object capability. The Mid-Infrared Instrument (MIRI) has both a camera and a spectrograph covering the wavelength range of 5 to 28 microns $[4,5,6,7$, 8, 9, 10, 11]. MIRI has a set of coronagraphs: the traditional Lyot and the Four Quadrant Phase Masks ones. The Fine Guidance Sensor (FGS) allows JWST to point precisely, so that it can obtain high-quality images. The Near Infrared Imager and Slit-less Spectrograph (NIRISS) part of the FGS has a wavelength range of 0.8 to 5.0 microns. The capabilities of the main instruments onboard JWST are summarised in the Figure 4. The photometric and spectroscopic performance of the instruments, beating HST and Spitzer is shown in the panels of the Figure 4. The telescope is sensitive enough to detect the heat radiated from a bee flying on the distance from the Earth to the Moon. The sensitivity plot is in the Figure 5 [12].

Most of the instrument are passively cooled to $\approx 40 \mathrm{~K}$ by the sun shield, except MIRI cooled to $7 \mathrm{~K}$. The sun shield has been designed to have 5 layers made of heat-resistant Kapton coated with silicon on the sun side and aluminium on the other surfaces. Thus, the sun side reaches $358 \mathrm{~K}$ $\left(85^{\circ} \mathrm{C}\right)$, while the dark side stays at $40 \mathrm{~K}\left(-233^{\circ} \mathrm{C}\right)$.

JWST capabilities includes imaging, spectroscopy, multi-object spectroscopy, slit-less spectroscopy, integrated field unit spectroscopy, single object coronography and aperture mask interferometry. There is a possibility to define parallel observations with combining two instruments simultaneously with a properly chosen dither pattern, the only limitation is the link band width and the size of the on-board buffer when submitting the data to the Earth. 


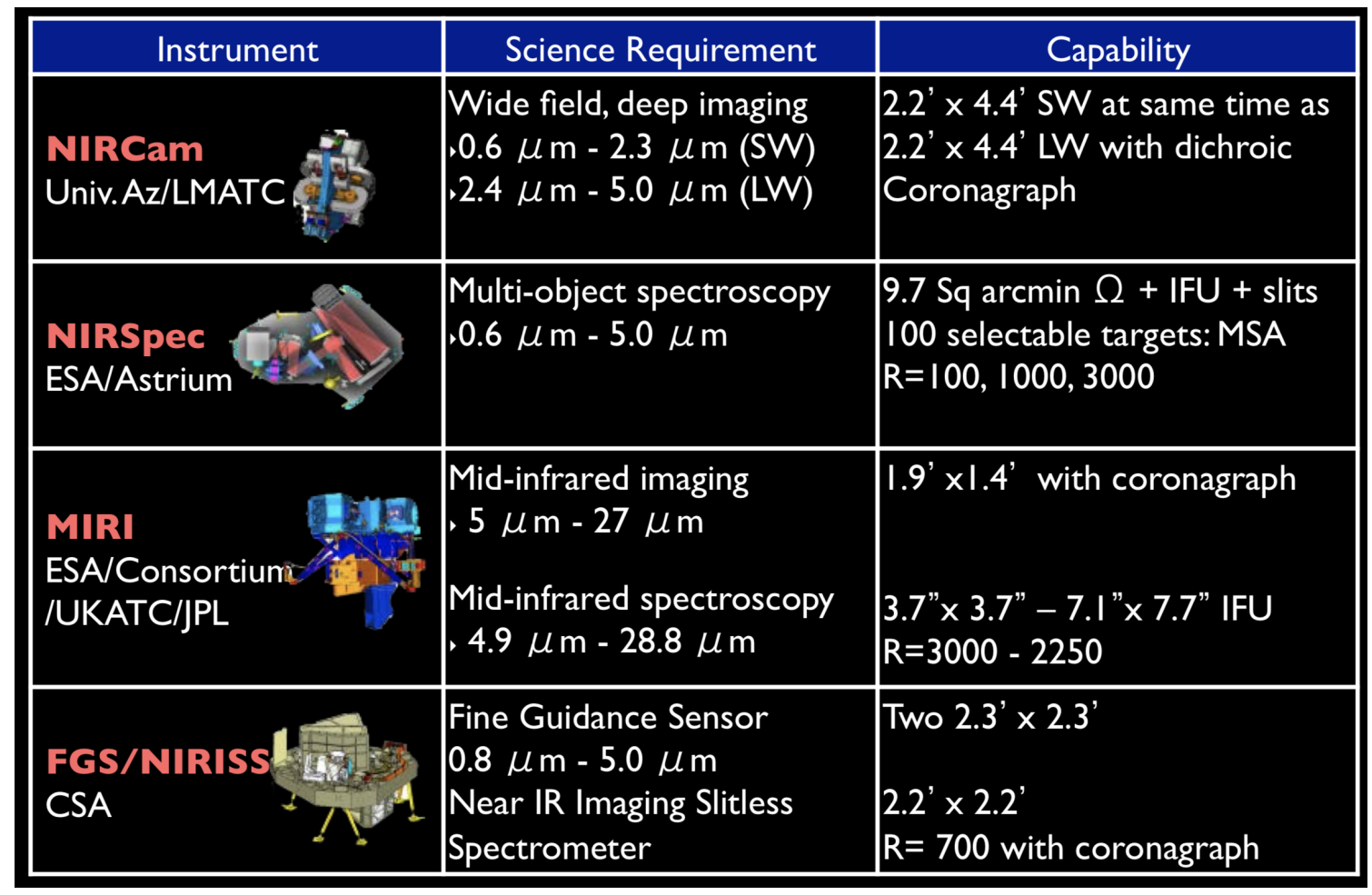

Figure 4: A summary of the capabilities of the JWST main scientific instruments. Credit: NASA.

\section{Scientific Goals}

Four essential astrophysical fields have been selected as the main objectives of the mission, described in great details in [13, 14]:

Birth of stars and protoplanetary systems - How do clouds of gas and dust collapse to form stars? How do planetary systems form?

Assembly of galaxies, the formation and evolution of galaxies - What are the first galaxies like? How are galaxies formed? How do the central black holes in galaxies influence their host galaxies? What happens when small and large galaxies collide or join together?

The end of the dark ages, the first light and re-ionisation - When and how does the reionisation occur? What sources caused it?

Exoplanets and planetary systems (including our solar system) and the origins of life How are the building blocks of planets assembled? What are the constituents of circumstellar disks that give rise to planetary systems? Do planets in a planetary system form in place, or travel inwards after forming in the outer reaches of the system? Can we find planets orbiting in the habitable zones of stars where it is possible for water, or perhaps life, to exist? Was there ever life on Mars?

Being more than one order of magnitude sensitive in some bands than any other observatory, a lot of unpredicted discoveries are expected.

The first half year is of the operation will be dedicated to commissioning and calibration of the instruments. The data acquired during this period will be released for public immediately in the Early Release Science (ERS) programme. 


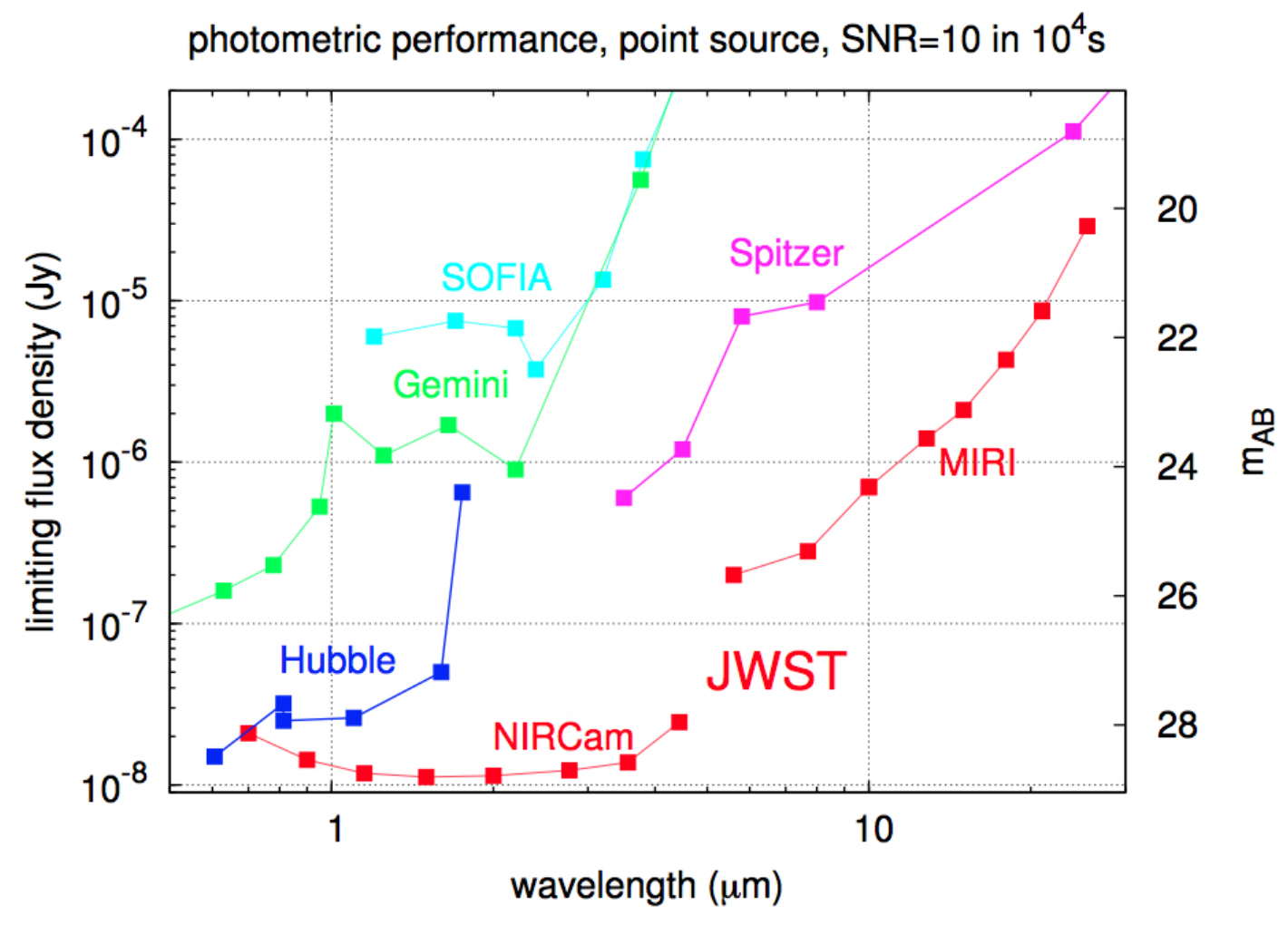

Figure 5: Comparison of sensitivity of the JWST instruments with existing missions. Credit: NASA.

\section{Highlighted Contribution}

From the vast ocean of proposals for observations, here the attention is drawn to three of them:

a) The JWST Ultra-Deep Field (UDF) is planned to go beyond the limits reached by the HST in projects, spanning the redshifts $z \sim 5-10$. This will allow the scientists to look for the most distant intermediate mass objects in the $z>10$. The UDF will lead to determination of the Star Formation History for the galaxies with $z \geq 6$. Thanks to the instrument sensitivity it can detect strong $\mathrm{H}_{\alpha}(z \sim 6.6-8.4)$ and [OIII] $(z \sim 8.9-11.3)$ emitters. By the UDF, JWST can constrain the Galaxy Stellar Mass Function and Cosmic Stellar Density for $z \geq 6$ galaxies. The imager will determine the morphology of the old stellar populations in the $\mathrm{z} \sim 4-6$ galaxies. The plan is to use combined NIRCam and MIRI instruments in parallel with dithering over the GOODS-S (also surveyed by Spitzer and HST) field which yields $255 \mathrm{sq}$. arcmin at 25h, 40h, 60h exposure times to benefit from the full wavelength range of the telescope.

b) Another brave proposal is the aim to observe a signal from a single Population III stars and their BH accretion disks at the very large redshifts $z \sim 7-17$, gravitationally lensed by a galaxy cluster. Typical caustic magnifications can be $\simeq 10^{4}-10^{5}$ for cluster transverse velocities of $\sim$ $1000 \mathrm{~km} \mathrm{~s}^{-1}$. Depending on BH masses, accretion-disk radii and feeding efficiencies, stellar-mass BH accretion-disk caustic transits could outnumber those from Population III stars. To observe Population III caustic transits directly may require to monitor $3-30$ lensing clusters to $A B \leq$ 
29 mag over a decade [21]. So far, discovered in a similar fashion, the record bearer is a HST observation of the most distant star lensed by a cluster, Icarus MACS J1149+2223 Lensed Star 1, at the redshift of $z=1.5$ [22].

c) The most promising theory for a short gamma-ray burst (sGRB) proposed so far is that the progenitor is a binary neutron star (NS) merger. A breakthrough in this theory was a recent direct observation of the gravitational waves GW170817 detected by the LIGO-VIRGO experiment matching the template of a neutron star merger and coinciding with a sGRB counterpart detected by the FERMI satellite [23]. Moreover, a near and/or far infra-red counterpart of a sGRB, called a kilonova, proposed in theory [24], has been observed later on [25]. The emission is believed to be a product of the r-process in the neutron-rich environment around the apocalyptic NS merger. Unlike the collimated gamma-ray emission lasting for an eye-blink, the kilonova emission is expected to be spherical and may last for days. JWST is capable to obtain a spectrum of such source in great details to further prove the sGRB-kilonova scenario and to inspect the spectral features and their evolution. A call for a Target of Opportunity (ToO) for precisely localised sGRB burst with a detected afterglow and/or a neutron star merger with a detected and well localised afterglow has been submitted in cases the gamma-ray burst occurs in the reach of the MIRI instrument at $\sim 5-10$ micron, as simulated in [26]. The coincidence positive gravitational wave detection is not necessary in this ToO.

\section{JWST Software}

There are several software tools being developed to make the life of a JWST scientist easier. The extensive user documentation is accessible [16]. There-in you can find links to the Exposure Time Calculator (ETC) and Astronomers Proposal Tool (APT) that are ready to use for planning the observations $[17,18]$. For advanced end-to-end simulations of the observations with the MIRI instrument, the MIRISim software package has been developed [19, 20]. MIRISim allows users to see a simulated datacube as the result of observing the sources on the sky predefined by a user through the MIRI detector. A user can customise the virtual observation defined by a variety of parameters such as the customised input sky scene, dither patterns, number of integrations, etc. An example of the workflow using MIRISim is shown in the Figure 6.

\section{Delay of the Launch Date}

The least favourite and the most discussed aspect of the JWST mission is a further heavy delay of its launch that has been announced recently. The Table 1 summarises the delays and the cost increase, starting from the initial cost and launch date estimate in 1997 up to today's situation. The main reason for the latest delay, communicated by the NASA headquarters, is the unsatisfactory results of vibration tests that lead to a partial redesign of the sun shield, the choice of the materials and improving its unfolding mechanism. The NASA strategy is clear that it is better to spend some extra time in testing rather than losing the entire spacecraft since parking the satellite around the L2 point makes any service or repair mission impossible. To this date, the launch of the rocket Ariane 5 with the JWST folded in the cargo top of the rocket has been scheduled for March 2021. 

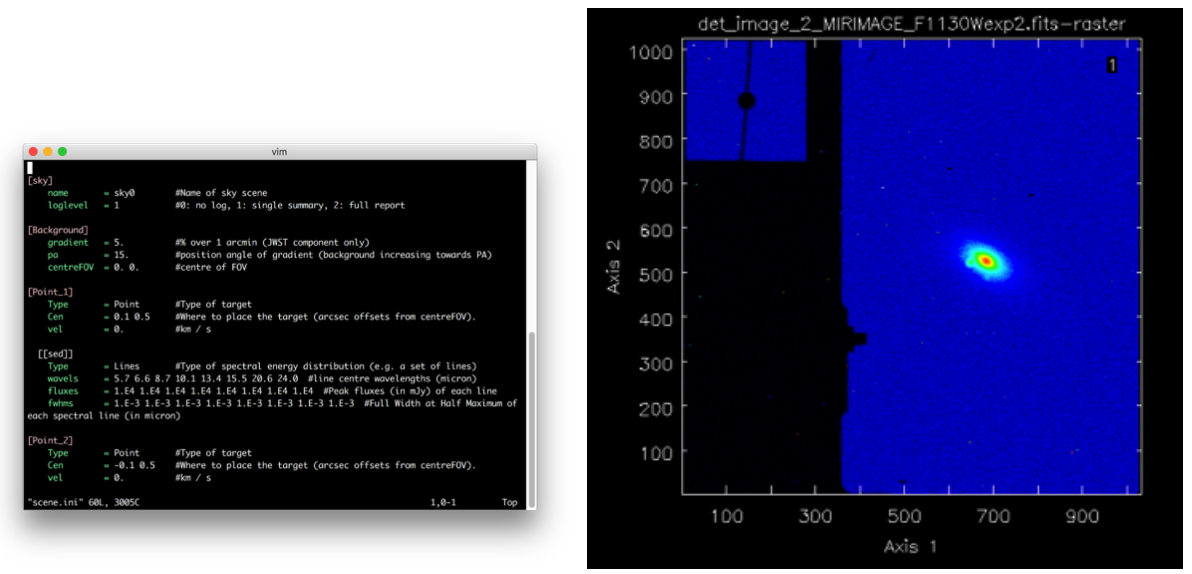

Figure 6: An example of MIRISim in action, starting from a scene description in a text file (left) to a simulated output image on the detector (right). Credit: Pamela Klaassen on behalf of the MIRISim team, private communication.

\begin{tabular}{ccc} 
Year & Launch & Budget Plan (Billion USD) \\
\hline 1997 & 2007 & 0.5 \\
1998 & 2007 & 1 \\
1999 & 2007 to 2008 & 1 \\
2000 & 2009 & 1.8 \\
2002 & 2010 & 2.5 \\
2003 & 2011 & 2.5 \\
2005 & 2013 & 3 \\
2006 & 2014 & 4.5 \\
2008 & 2014 & 5.1 \\
2010 & 2015 to 2016 & 6.5 \\
2011 & 2018 & 8.7 \\
2013 & 2018 & 8.8 \\
2017 & 2019 & 8.8 \\
2018 & 2020 & $\geq 8.8$ \\
2018 & 2021 & $\geq 10$
\end{tabular}

Table 1: The estimated launch dates and the expected costs of JWST. Credit: Wiki.

\section{Conclusion}

All instruments are now fully integrated with the telescope and they are performing to the specs. A detailed review of every single component has been planned to diminish any flaw at or after the launch. The reasonable launch date, currently estimated today, is March 2021.

If things go well, the JWST telescope will dominate the IR astronomy domain in the next decade thanks to its unique outstanding capabilities. It will bring answers to many fundamental questions on early star and galaxy formation in the Universe, uncover the composition of exoplanets and for sure, it will also bring many new unexpected discoveries. Fingers crossed that all the 
construction issues would be solved and JWST would be launched and deployed successfully and achieve many great new discoveries!

\section{References}

[1] Clampin, M. 2008, Advances in Space Research, Vol. 41, 1983

[2] https://www.jwst.nasa.gov

[3] https://jwst.stsci.edu/instrumentation

[4] Rieke, G. H., Wright, G. S., Böker, T., et al. 2015, PASP, Vol. 127, 584

[5] Wright, G. S., Wright, D., Goodson, G. B., et al. 2015, PASP, Vol. 127, 595

[6] Bouchet, P., García-Marín, M., Lagage, P.-O., et al. 2015, PASP, Vol. 127, 612

[7] Kendrew, S., Scheithauer, S., Bouchet, P., et al. 2015, PASP, Vol. 127, 623

[8] Boccaletti, A., Lagage, P.-O., Baudoz, P., et al. 2015, PASP, Vol. 127, 633

[9] Wells, M., Pel, J.-W., Glasse, A., et al. 2015, PASP, Vol. 127, 646

[10] Rieke, G. H., Ressler, M. E., Morrison, J. E., et al. 2015, PASP, Vol. 127, 665

[11] Ressler, M. E., Sukhatme, K. G., Franklin, B. R., et al. 2015, PASP, Vol. 127, 675

[12] Glasse, A., Rieke, G. H., Bauwens, E., et al., 2015, PASP, Vol. 127, 686

[13] Gardner et al., 2006, Space Science Reviews, Vol. 123, 485

[14] Kalirai, J. 2018, Contemporary Physics, 59, 251

[15] Rieke G. H. et al., 2015, The Astronomical Society of the Pacific, Vol. 127, N953

[16] http://docs.jwst.nasa.gov

[17] https://jwst.etc.stsci.edu

[18] http://www.stsci.edu/hst/proposing/apt

[19] https://miricle.org

[20] Gordon, K. D., Chen, C. H., Anderson, R. E., et al. 2015, PASP, 127, 696

[21] Windhorst, R. et al., 2018, American Astronomical Society Meeting Abstracts, Vol. 232, 325

[22] Miralda-Escude, J., 1991, The Astrophysical Journal, Vol. 379, 94-98

[23] Abbott, B. P. et al., 2017, The Astrophysical Journal, Vol. 848, L13

[24] Metzger, B. D. \& Berger, E., 2012, The Astrophysical Journal, Vol. 746, 48

[25] Cowperthwaite, P. S. et al. 2017, The Astrophysical Journal Letters, Vol. 848, L17

[26] Bartos, I., Huard, T. L. and Marka, S., 2015, The Astrophysical Journal, Vol. 816, 61 